\title{
Light-Meson Spectroscopy with COMPASS
}

\author{
Boris Grube* for the COMPASS Collaboration \\ Physik-Department E18 \\ Technische Universität München \\ James-Franck-Str. \\ D-85748 Garching \\ Germany \\ E-mail: bgrube@ph.tum.de
}

COMPASS is a multi-purpose fixed-target experiment at the CERN Super Proton Synchrotron investigating the structure and spectrum of hadrons. One primary goal is the search for new hadronic states, in particular spin-exotic mesons and glueballs. After a short pilot run in 2004 with a $190 \mathrm{GeV} / c \pi^{-}$beam on a $\mathrm{Pb}$ target, which showed a significant spin-exotic $J^{P C}=1^{-+}$ resonance consistent with the controversial $\pi_{1}(1600)$, COMPASS collected large data samples with negative and positive hadron beams on $\mathrm{H}_{2}, \mathrm{Ni}, \mathrm{W}$, and $\mathrm{Pb}$ targets in 2008 and 2009. We present results from a partial-wave analysis of diffractive dissociation of $190 \mathrm{GeV} / c \pi^{-}$into $\pi^{-} \pi^{+} \pi^{-}$final states on $\mathrm{Pb}$ and $\mathrm{H}_{2}$ targets with squared four-momentum transfer in the range $0.1<t^{\prime}<1(\mathrm{GeV} / c)^{2}$. This reaction provides clean access to the light-quark meson spectrum up to masses of $2.5 \mathrm{GeV} / \mathrm{c}^{2}$. A first comparison of the data from $\mathrm{Pb}$ and $\mathrm{H}_{2}$ target shows a strong target dependence of the production strength of states with spin projections $M=0$ and 1 relative to the $a_{2}(1320)$. The $2004 \mathrm{~Pb}$ data were also analyzed in the region of small squared fourmomentum transfer $t^{\prime}<10^{-2}(\mathrm{GeV} / c)^{2}$, where we observe interference of diffractive production and photoproduction in the Coulomb-field of the $\mathrm{Pb}$ nucleus.

The Xth Nicola Cabibbo International Conference on Heavy Quarks and Leptons,

October 11-15, 2010

Frascati (Rome) Italy

\footnotetext{
*Speaker.
} 


\section{Introduction}

The COmmon Muon and Proton Apparatus for Structure and Spectroscopy (COMPASS) [1] is a fixed-target experiment at the CERN Super Proton Synchrotron (SPS). It is a two-stage spectrometer that covers a wide range of scattering angles and particle momenta with high angular resolution. It is equipped with hadronic and electromagnetic calorimeters so that COMPASS can reconstruct final states with charged as well as neutral particles. The target is surrounded by a Recoil Proton Detector (RPD) that measures the time of flight of the recoil protons. COMPASS uses the M2 beam line of the SPS which can deliver secondary hadron beams with a momentum of up to $300 \mathrm{GeV} / c$ and a maximum intensity of $5 \cdot 10^{7} \mathrm{~s}^{-1}$. The negative hadron beam consists of $96.0 \% \pi^{-}, 3.5 \% K^{-}$, and $0.5 \% \bar{p}$. Two ChErenkov Differential counters with Achromatic Ring focus (CEDAR) upstream of the target are used to identify the incoming beam particles.

Its large acceptance, high resolution, and high-rate capability make the COMPASS experiment an excellent device to study the spectrum of light mesons in diffractive and central production up to masses of about $2.5 \mathrm{GeV} / c^{2}$. Since COMPASS is able to measure final states with charged as well as neutral particles, resonances can be studied in many different reactions and decay channels.

During a pilot run in 2004 and subsequent data taking periods in 2008 and 2009 COMPASS has acquired large data sets of diffractive dissociation of $190 \mathrm{GeV} / c \pi^{-}$on $\mathrm{H}_{2}, \mathrm{Ni}, \mathrm{W}$, and $\mathrm{Pb}$ targets. In these events the beam pion is excited to some resonance $X^{-}$via $t$-channel Reggeon exchange with the target (cf. Fig. 1). At $190 \mathrm{GeV} / c$ the process is dominated by Pomeron exchange, so that isospin and $G$-parity of the intermediate state $X^{-}$are that of the beam pion.

In 2004 the trigger selected one incoming and at least two outgoing charged particles, whereas in 2008 a signal from the recoil proton was required in the RPD. In the offline event selection diffractive events were enriched by an exclusivity cut of $\pm 4 \mathrm{GeV}$ around the nominal beam energy.

Diffractive reactions are known to exhibit a rich spectrum of produced states and are characterized by two kinematic variables: the square of the total center-of-mass energy and the squared four-momentum transfer from the incoming beam particle to the target, $t=\left(p_{\text {beam }}-p_{X}\right)^{2}$. It is

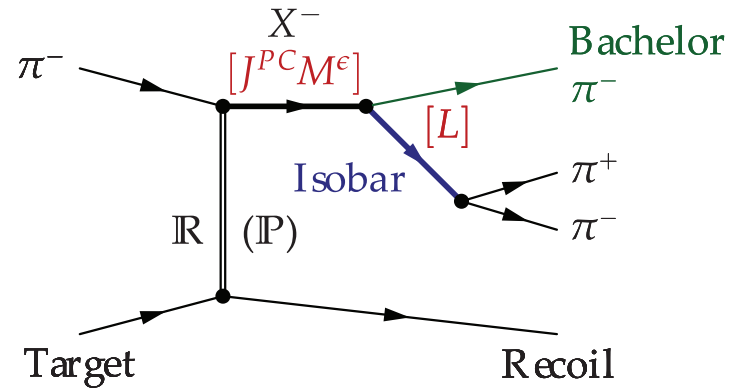

Figure 1: Diffractive production of a resonance $X^{-}$ via $t$-channel Reggeon exchange and its decay into the $\pi^{-} \pi^{+} \pi^{-}$final state as described in the isobar model.

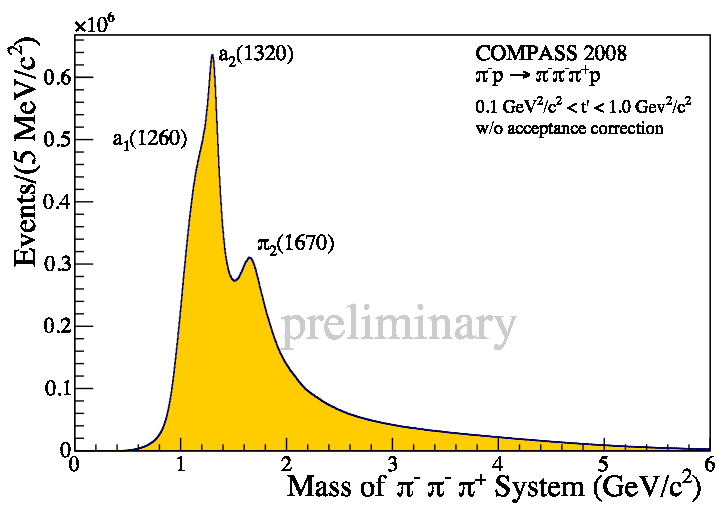

Figure 2: $\pi^{-} \pi^{+} \pi^{-}$invariant mass distribution of the 2008 data sample on $\mathrm{H}_{2}$ target with $0.1<t^{\prime}<$ $1.0(\mathrm{GeV} / c)^{2}$. 


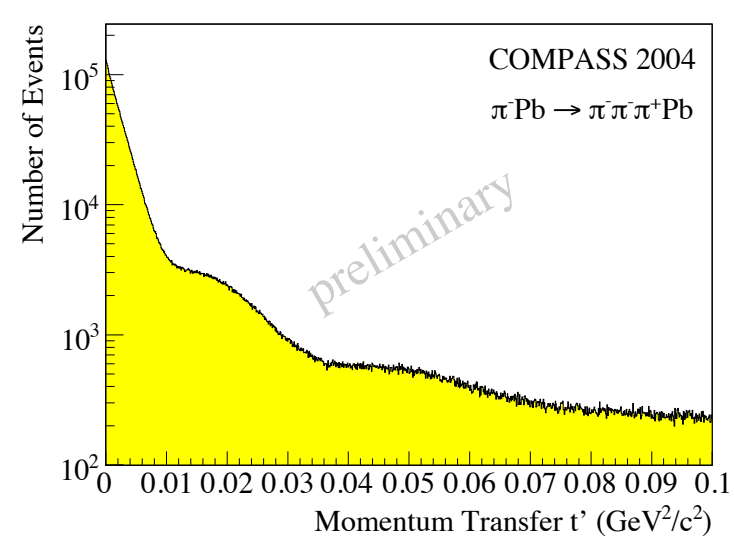

Figure 3: $t^{\prime}$ spectrum of the 2004 data on a $\mathrm{Pb}$ target: At very small momentum transfer of $t^{\prime}<$ $10^{-3}(\mathrm{GeV} / c)^{2}$ (not visible in this plot) photoproduction in the Coulomb field of the target nucleus contributes. For $t^{\prime}$ up to about $10^{-2}(\mathrm{GeV} / c)^{2}$ the spectrum can be described by an exponential distribution. At larger $t^{\prime}$ the data exhibit a diffraction pattern.

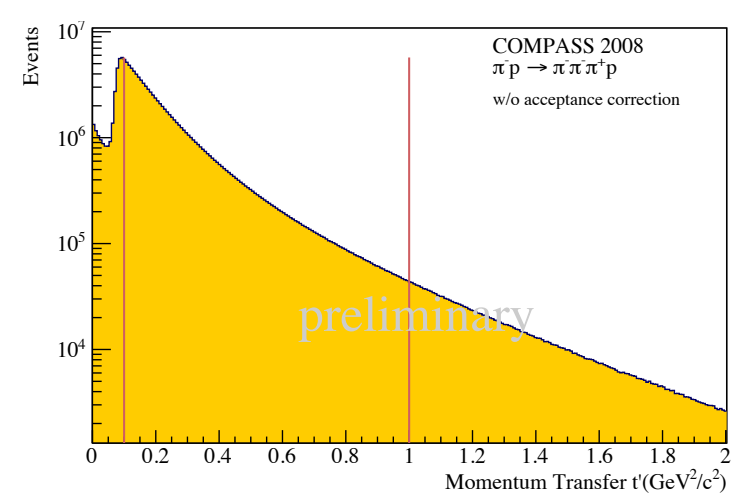

Figure 4: $t^{\prime}$ distribution of the $2008 \mathrm{H}_{2}$ data: The data were recorded by triggering on the recoil proton so that events with $t^{\prime}$ below about $0.1(\mathrm{GeV} / c)^{2}$ are suppressed.

customary to use the variable $t^{\prime}=|t|-|t|_{\text {min }}$ instead of $t$, where $|t|_{\min }$ is the minimum value of $|t|$ for a certain three-pion invariant mass. Figures 3 and 4 show the $t^{\prime}$ distributions of the 2004 data sample with a $\mathrm{Pb}$ target and that of the 2008 data on $\mathrm{H}_{2}$, respectively.

Figure 2 shows the $\pi^{-} \pi^{+} \pi^{-}$invariant mass distribution of the 2008 data sample. It exhibits clear structures in the mass regions of the well-known resonances $a_{1}(1260), a_{2}(1320)$, and $\pi_{2}(1670)$. In order to find and disentangle the various resonances in the data, a Partial-Wave Analysis (PWA) was performed. In the PWA the isobar model [3] is used to decompose the decay $X^{-} \rightarrow \pi^{-} \pi^{+} \pi^{-}$into a chain of successive two-body decays as shown in Fig. 1: The $X^{-}$with quantum numbers $J^{P C}$ and spin projection $M^{\varepsilon}$ decays into a di-pion resonance, the so-called isobar, and a bachelor pion. The isobar has spin $S$ and a relative orbital angular momentum $L$ with respect to

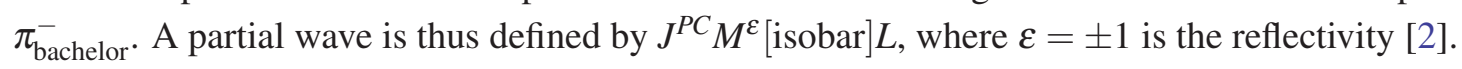

The production amplitudes are determined by extended maximum likelihood fits performed in $40 \mathrm{MeV} / c^{2}$ wide bins of the three-pion invariant mass $m_{X}$. In these fits no assumption is made on the produced resonances $X^{-}$other then that their production strengths are constant within a $m_{X}$ bin. The PWA model includes five $\pi^{+} \pi^{-}$isobars [4]: $(\pi \pi)_{S \text {-wave }}, \rho(770), f_{0}(980), f_{2}(1270)$, and $\rho_{3}(1690)$. They were described using relativistic Breit-Wigner line shape functions including Blatt-Weisskopf barrier penetration factors [5]. For the $\pi^{+} \pi^{-} S$-wave we use the parameterization from [6] with the $f_{0}(980)$ subtracted from the elastic $\pi \pi$ amplitude and added as a separate BreitWigner resonance. 


\section{PWA in Large-Momentum-Transfer Region}

The PWA model for the squared four-momentum transfer region $0.1<t^{\prime}<1(\mathrm{GeV} / c)^{2}$ (cf. Fig. 4) consists of 41 partial waves with $J \leq 4$ and $M \leq 1$ plus one incoherent isotropic background wave. Because in the chosen $t^{\prime}$ range the beam predominantly scatters off the individual nucleons in the target nucleus, a rank-two spin-density matrix is used. This accounts for spin-flip and spinnon-flip amplitudes at the target vertex. In order to describe the data, mostly positive reflectivity waves are needed which corresponds to production with natural parity exchange.

The three main waves $1^{++} 0^{+}[\rho \pi] S, 2^{++} 1^{+}[\rho \pi] D$, and $2^{-+} 0^{+}\left[f_{2} \pi\right] S$ contain resonant structures that correspond to the $a_{1}(1260), a_{2}(1320)$, and $\pi_{2}(1670)$, respectively. The resonance parameters extracted from the $2004 \mathrm{~Pb}$ data are in good agreement with the PDG values [4]. In addition the data exhibit a resonant peak around $1660 \mathrm{MeV} / c^{2}$ in the spin-exotic $1^{-+} 1^{+}[\rho \pi] P$ wave that is consistent with the disputed $\pi_{1}(1600)$ [4].

A first comparison of the $2008 \mathrm{H}_{2}$ data with the $2004 \mathrm{~Pb}$ data without acceptance corrections shows a surprisingly large dependence on the target material. The two data sets are normalized to the narrow $a_{2}(1320)$ resonance in the $2^{++} 1^{+}[\rho \pi] D$ wave. The $\mathrm{H}_{2}$ data exhibit a strong suppression of $M=1$ waves relative to the $a_{2}(1320)$, whereas the corresponding $M=0$ waves are enhanced such that the intensity sum over $M$ remains about the same for both target materials. Figure 5 shows this effect for the $a_{1}(1260)$ peak in the $J^{P C}=1^{++}$waves. This will be studied further using the data taken with $\mathrm{Ni}$ and $\mathrm{W}$ targets.

\section{PWA in Small-Momentum-Transfer Region}

In the small-momentum-transfer region $t^{\prime}<10^{-2}(\mathrm{GeV} / \mathrm{c})^{2}$ (cf. Fig. 3) two production mechanisms contribute: At impact parameters larger than the radius of the target nucleus, which corresponds to very low $t^{\prime}$, electromagnetic interactions dominate. In these processes the incoming beam pions scatter off the quasi-real virtual photons that surround the heavy target nucleus [7]. For $t^{\prime}$ larger than about $10^{-3}(\mathrm{GeV} / c)^{2}$ the strong interaction in the form of Pomeron exchange becomes dominant.

The data are fitted using a PWA model that consists of 37 waves (plus an incoherent isotropic background wave) and a rank-two spin-density matrix. In a first analysis the $t^{\prime}$ range was further subdivided into a "low- $t^{\prime \prime}$ " region with $1.5 \cdot 10^{-3}<t^{\prime}<10^{-2}(\mathrm{GeV} / c)^{2}$ and a "Primakoff" region with $t^{\prime}<0.5 \cdot 10^{-3}(\mathrm{GeV} / c)^{2}$. The low- $t^{\prime}$ region is dominated by diffractive production processes, whereas in the Primakoff region also photoproduction contributes. This effect is enhanced for waves with spin projection $M=1$, because the diffractive production strength is proportional to $\left(t^{\prime}\right)^{M} \exp \left(-b t^{\prime}\right)$. Figure 6 shows the behavior of the two main waves: the $1^{++} 0^{+}[\rho \pi] S$ wave, which mainly consists of the $a_{1}(1260)$ resonance, has $M=0$ so that diffractive production is still dominant in the Primakoff region. The $a_{2}(1320)$ resonance in the $2^{++} 1^{+}[\rho \pi] D$ wave, however, has $M=1$ so that in the Primakoff region diffractive production of this resonance is suppressed whereas photoproduction is enhanced. As Fig. 6 (center) shows, the $t^{\prime}$ bins were chosen such that the number of $a_{2}(1320)$ in the Primakoff region is similar to that in the low- $t^{\prime}$ region. The phase motion of the narrow $a_{2}(1320)$ in the large-mass tail of the $a_{1}(1260)$ is similar in both $t^{\prime}$ regions 

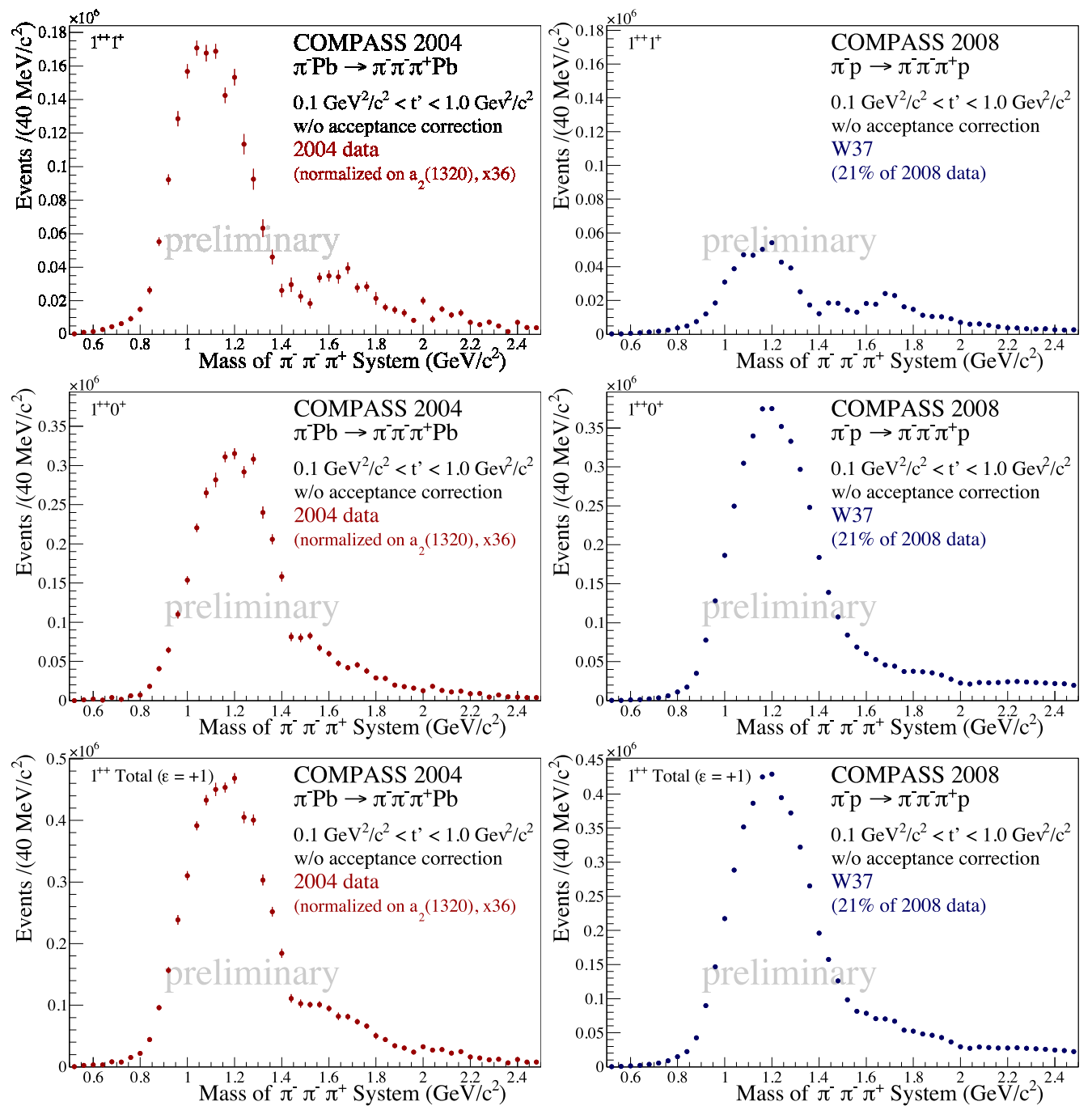

Figure 5: Normalized intensity sums of the $J^{P C}=1^{++}$partial waves for spin projection quantum numbers $M=1$ (top) and $M=0$ (middle). The sum over all $M$ is shown in the bottom plots. The left column shows 2004 data with $\mathrm{Pb}$ target, the right column 2008 data on $\mathrm{H}_{2}$ target. The wave intensities are dominated by a broad structure around $1.2 \mathrm{GeV} / c^{2}$ which is the $a_{1}(1260)$.

except for an offset of about $90^{\circ}$ (cf. Fig. 6 right). This is due to the different production phases of the $a_{2}$ (1320) introduced by photon and Pomeron exchange, respectively.

In order to study the transition between the two $a_{2}(1320)$ production mechanisms, a PWA in bins of $t^{\prime}$ was performed in a wide mass region around the $a_{2}(1320)$ (see Fig. 7). The resulting $t^{\prime}$ spectrum of the $a_{1}(1260)$ has a slope compatible with diffractive production (cf. Fig. 3), whereas the $a_{2}(1320)$ spectrum features a steep rise at low $t^{\prime}$ as expected for photoproduction, given the finite experimental resolution. The phase difference of the $a_{2}(1320)$ and the $a_{1}(1260)$ vanishes at larger $t^{\prime}$ indicating that both resonances are produced diffractively. The $t^{\prime}$ dependence of the relative phase is roughly described by a Glauber model analogous to [8]. Further analyses will focus on the study of the reaction $\pi \gamma \rightarrow 3 \pi$ close to threshold in order to test recent $\chi$ PT calculations [9]. 

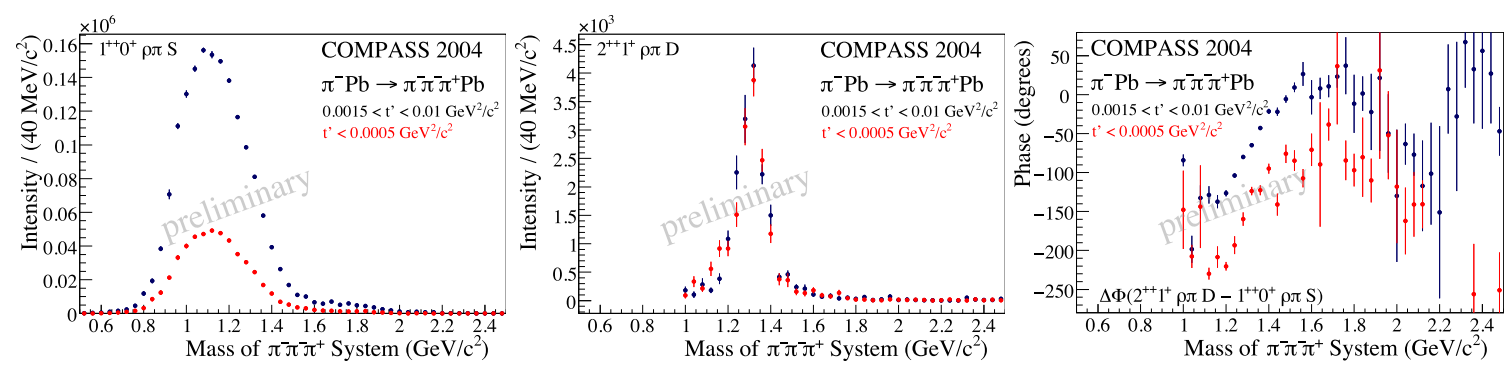

Figure 6: Intensities of the $1^{++} 0^{+}[\rho \pi] S$ (left) and $2^{++} 1^{+}[\rho \pi] D$ waves (center) plus their phase difference (right) as a function of the $\pi^{-} \pi^{+} \pi^{-}$mass in two bins of the squared four-momentum transfer $t^{\prime}$ : Blue points represent the "low- $t^{\prime}$ " region $1.5 \cdot 10^{-3}<t^{\prime}<10^{-2}(\mathrm{GeV} / c)^{2}$, red points the "Primakoff" region $t^{\prime}<0.5 \cdot 10^{-3}(\mathrm{GeV} / c)^{2}$.
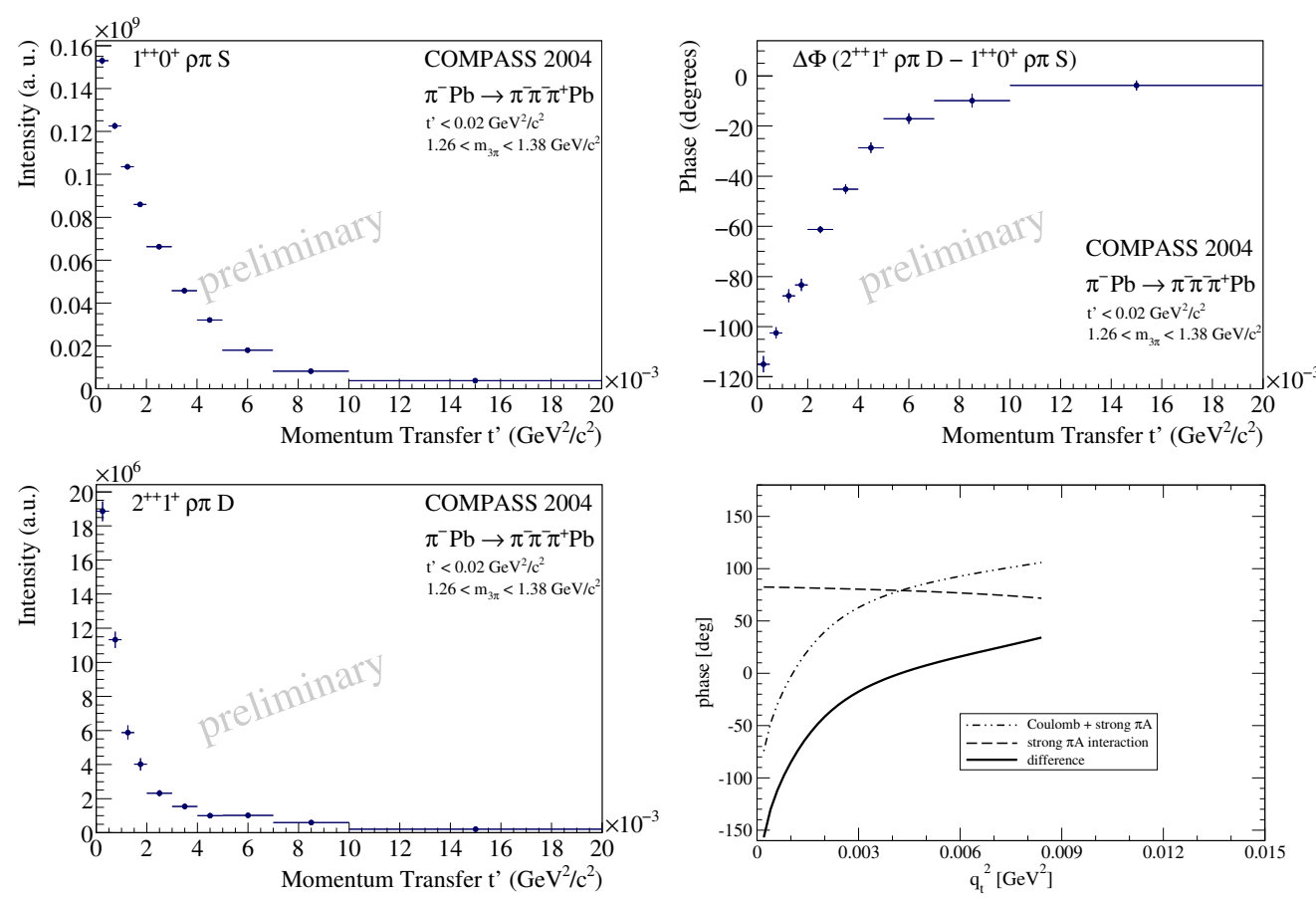

Figure 7: Intensities of the $1^{++} 0^{+}[\rho \pi] S$ (top-left) and $2^{++} 1^{+}[\rho \pi] D$ waves (bottom-left) plus their relative phase (top-right) as a function of the squared four-momentum transfer $t^{\prime}$ in a mass range around the $a_{2}(1320)$ mass. The phase difference between the strong $\pi A$ interaction and the Coulomb interaction as predicted by a Glauber model calculation is shown in the lower right plot.

\section{Acknowledgments}

This work is supported by the German Bundesministerium für Bildung und Forschung BMBF, the Maier-Leibnitz-Labor der LMU und TU München, the DFG Cluster of Excellence Origin and Structure of the Universe, and CERN-RFBR grant 08-02-91009. 


\section{References}

[1] P. Abbon et al., Nucl. Instrum. Meth. A577, 455 (2007).

[2] S. U. Chung and T. L. Trueman, Phys. Rev. D11 633, (1975).

[3] J. D. Hansen et al., Nucl. Phys. B81, 403 (1974).

[4] M. G. Alekseev et al. [COMPASS Collaboration], Phys. Rev. Lett. 104, 241803 (2010).

[5] F. von Hippel and C. Quigg, Phys. Rev. D5, 624 (1972).

[6] D.V. Amelin et al., Phys. Lett. B356, 595 (1995).

[7] I. Y. Pomeranchuk and I. M. Shmushkevich, Nucl. Phys. 23, 452 (1961).

[8] G. Fäldt and U. Tengblad, Phys. Rev. C79, 014607 (2009).

[9] N. Kaiser and J. M. Friedrich, Eur. Phys. J. A36, 181 (2008); N. Kaiser, Nucl. Phys. A848, 198 (2010). 